\title{
EFEITO DO AMBIENTE PROTEGIDO CULTIVADO COM MELÃO SOBRE OS ELEMENTOS METEOROLÓGICOS E SUA RELAÇÃO COM AS CONDIÇÕES EXTERNAS ${ }^{1}$
}

\author{
MANUEL A. N. VÁSQUEZ ${ }^{2}$, MARCOS V. FOLEGATTI ${ }^{3}$, NILDO DA S. DIAS ${ }^{4}$, \\ CLÁUDIO R. DA SILVA
}

\begin{abstract}
RESUMO: O objetivo deste trabalho foi estudar o efeito do ambiente protegido cultivado com melão (Cucumis melo L.) sobre os elementos meteorológicos e sua relação com as condições externas. $\mathrm{O}$ experimento foi conduzido no período de 5-10-2001 a 7-1-2002, em estufa plástica de $420 \mathrm{~m}^{2}$, construída com estrutura metálica galvanizada, com dois vãos de altura central de 4,6 m e lateral de $3,0 \mathrm{~m}$, composto de quatro janelas frontais, cobertas com filme de polietileno transparente de alta densidade, com aditivo ultravioleta e espessura de $150 \mu \mathrm{m}$. A irrigação foi efetuada por gotejamento, com lâmina total de 279,6 mm, manejada utilizando um minitanque instalado no interior do ambiente. Os elementos meteorológicos foram obtidos por sistema de aquisição automático instalado no interior do ambiente protegido e, externamente, com medidas da radiação global, temperatura e umidade relativa do ar. Verificou-se que a radiação solar global e a umidade relativa no ambiente protegido foram, em média, inferiores às condições externas, enquanto a temperatura foi superior no período analisado. A relação entre os elementos meteorológicos medidos externamente e no interior do ambiente protegido foi expressa por meio de equações de regressão linear e quadrática.
\end{abstract}

PALAVRAS-CHAVE: radiação, evapotranspiração, irrigação, Cucumis melo L.

\section{EFFECT OF GREENHOUSE CULTIVATED WITH MELON CROP UNDER METEOROLOGICAL ELEMENTS AND ITS RELATIONSHIP WITH EXTERNAL CONDITIONS}

\begin{abstract}
The objective of this research was to study the effect of greenhouse cultivated with melon (Cucumis melo L.) under meteorological elements and its relationship with external conditions. The study was carried out during the period of October 5, 2001 to January 7, 2002. The greenhouse had $420 \mathrm{~m}^{2}$, constructed in galvanized metallic structure with two voids, height in the central part of $4.6 \mathrm{~m}$ and lateral of $3.0 \mathrm{~m}$, having four front windows covered with a film of transparent polyethylene of high density with addictive ultraviolet and thickness of $150 \mu \mathrm{m}$. A drip irrigation system was used with a total water depth of $279.6 \mathrm{~mm}$ applied. The irrigation management was done through a small evaporation pan installed inside the greenhouse. The weather data were obtained through an automatic acquisition system placed inside and outside of the greenhouse with measurements of global solar radiation, temperature and humidity of the air. The solar radiation and relative humidity were lower in the greenhouse while the temperature was higher. The relationship between weather parameters inside and outside of the greenhouse, was expressed by linear and quadratic regression equations.
\end{abstract}

KEYWORDS: radiation, evapotranspiration, irrigation, Cucumis melo L.

\footnotetext{
${ }^{1}$ Extraído da tese de doutorado do primeiro autor. Financiado pela FAPESP.

${ }^{2}$ Doutorando em Agronomia, ESALQ/USP, LER, Piracicaba - SP, Fone: (0XX19) 429.4217, manvasqu@carpa.ciagri.br. Bolsista FAPESP.

${ }^{3}$ Professor Doutor,'ESALQ/USP, LER, Piracicaba - SP, mvfolega@esalq.usp.br.

${ }^{4,5}$ Doutorando em Agronomia, ESALQ/USP, LER, Piracicaba - SP. Bolsista CAPES.

Recebido pelo Conselho Editorial em: 6-2-2004

Aprovado pelo Conselho Editorial em: 31-3-2005
}

Eng. Agríc., Jaboticabal, v.25, n.1, p.137-143, jan./abr. 2005 


\section{INTRODUÇÃO}

Nas diversas regiões do Brasil, a utilização de ambiente protegido, principalmente para a produção de plantas ornamentais e hortícolas, tem aumentado consideravelmente, devido às vantagens relacionadas à maior proteção quanto aos fenômenos climáticos, como geadas, excesso de chuvas, queda acentuada de temperatura durante a noite, proteção do solo contra a lixiviação e redução dos custos com fertilizantes e defensivos. Além disso, as colheitas nesses ambientes excedem ostensivamente às que se obtêm a céu aberto (OLIVEIRA, 1995).

Segundo JONES (1992), a radiação solar é a principal fonte de energia para as plantas, sendo sua maior parte convertida em calor, impulsionando o processo de transpiração e também alterando a temperatura dos tecidos vegetais com conseqüência para a taxa dos processos metabólicos e o balanço entre eles. Em revisão feita por KIRDA (1994), verifica-se relação linear entre o consumo de água de uma cultura em ambiente protegido e a radiação solar diária.

Assim mesmo, o aquecimento das folhas aumenta o gradiente de pressão de vapor entre o dossel e o ambiente, enquanto a abertura dos estômatos reduz a resistência à transferência de vapor, podendo combinar uma estreita relação entre radiação, temperatura e umidade dentro das estufas, resultando, empiricamente, em relação linear entre transpiração e radiação (JONES, 1992).

Quando se compara o cultivo em ambiente protegido com o cultivo a céu aberto, verifica-se que a evapotranspiração é geralmente menor no interior do ambiente protegido do que externamente, atribuindo-se, basicamente, à parcial opacidade da cobertura plástica e à redução da ação dos ventos, que são os principais fatores da demanda evaporativa da atmosfera. Embora a temperatura e a umidade relativa do ar, em alguns momentos, podem atingir valores maiores no interior do ambiente protegido, com estreita dependência com as condições meteorológicas. Em geral, a evapotranspiração no interior do ambiente protegido fica em torno de $60-80 \%$ da verificada no exterior (FARIAS et al., 1993).

PEREIRA et al. (1997) manifestam que, quanto maior a deficiência hídrica, menor será a evaporação e, conseqüentemente, maior a energia disponível que será utilizada no aquecimento, resultando em grandes variações na temperatura.

$\mathrm{O}$ polietileno de baixa densidade (PEBD) é um material que apresenta boa transparência à radiação solar, deixando passar em média 70 a $80 \%$ e máximo de $95 \%$ da radiação solar (BURIOL et al., 1993).

SEEMAN (1979) afirma que a quantidade de energia solar não transmitida é função do tipo de cobertura plástica e do ângulo de incidência dos raios solares, que são condicionados pela orientação da cobertura e posição do Sol.

SCATOLINI (1996) relata maior efeito da cobertura plástica sobre as temperaturas máximas com valores de $1,2^{\circ} \mathrm{C}$ a $4,4{ }^{\circ} \mathrm{C}$ acima das observadas externamente. Com relação às temperaturas mínimas do ar, essas tendem a ser iguais ou ligeiramente superiores à observada externamente, sendo afetadas pelo manejo da ventilação das estufas durante o dia, por meio de abertura e fechamento de cortinas laterais (BURIOL et al., 1993).

Portanto, considerando que a cobertura plástica do ambiente protegido altera os elementos meteorológicos e impede a entrada de água de chuva, torna-se importante para o entendimento das exigências hídricas das plantas conhecer a amplitude dessas variações no ambiente protegido. Desse modo, teve-se o objetivo de comparar os elementos meteorológicos no interior do ambiente protegido cultivado com melão (Cucumis melo L.) e sua relação com as condições externas. 


\section{MATERIAL E MÉTODOS}

O experimento foi conduzido na área experimental do Departamento de Engenharia Rural da Escola Superior de Agricultura "Luiz de Queiroz" - USP, Piracicaba - SP, em ambiente protegido de

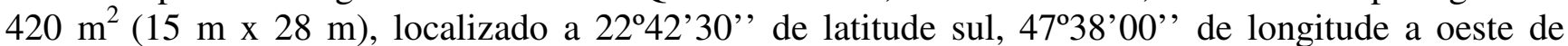
Greenwich e $576 \mathrm{~m}$ de altitude, no período de 5-10-2001 a 7-1-2002.

O ambiente protegido utilizado possuía dois vãos, com estrutura metálica galvanizada e altura na parte central dos vãos de 4,6 m e nas laterais de 3,0 m, constituído de quatro janelas frontais, cobertas com filme de polietileno transparente de alta densidade, com aditivo ultravioleta, e espessura de 150 $\mu \mathrm{m}$. As laterais da mesma foram fechadas com tela plástica branca tipo clarite $50 \%$, providas também de cortinas de polietileno transparente para regular as temperaturas e o fluxo de ar.

O solo foi classificado como Latossolo Vermelho-Amarelo, fase arenosa, denominada "Série Sertãozinho". A correção e a adubação do solo seguiram as recomendações de RAIJ et al. (1986) e KIEHL (1985). Os adubos foram aplicados via água de irrigação com frequiência de dois dias, à base de $150 \mathrm{~kg} \mathrm{ha}^{-1}$ de $\mathrm{N}$ e $400 \mathrm{~kg} \mathrm{ha}^{-1}$ de $\mathrm{K}_{2} \mathrm{O}$, nas formas de nitrato de amônia e nitrato de potássio, de acordo com a marcha de absorção da cultura durante seu ciclo, recomendado por BAR-YOSEF (1999).

Utilizaram-se sementes do híbrido de melão Bonus $\mathrm{n}^{\underline{0}}$ 2, tipo rendilhado, semeadas em tubos de polietileno com substrato comercial organo-mineral, plantadas em 5-10-2001, após 19 dias da semeadura, quando as plantas apresentavam duas folhas definitivas. Utilizaram-se covas de 3,6 m de comprimento $\mathrm{x}$ 0,6 $\mathrm{m}$ de largura, espaçadas a 0,5 m entre covas e 0,8 $\mathrm{m}$ entre ruas. Cada cova continha duas fileiras de plantas espaçadas 0,6 m entre plantas e 0,20 m entre fileiras, em esquema alternado, totalizando 22.286 plantas ha $^{-1}$.

O sistema de irrigação adotado foi o de gotejamento com reposição diária de $67 \%$ da lâmina evaporada de um minitanque evaporimétrico (dimensões de $0,60 \mathrm{~m}$ de diâmetro e $0,25 \mathrm{~m}$ de altura) localizado a 1/3 do comprimento do ambiente protegido. Os emissores na linha foram do tipo autocompensante, com vazão nominal de $1,6 \mathrm{~L} \mathrm{~h}^{-1}$, espaçados de $0,30 \mathrm{~m}$ e disposto no centro das duas fileiras de plantas e pressão nominal de 15 m.c.a.

Os elementos meteorológicos no interior do ambiente protegido foram obtidos por um sistema de aquisição automático de dados (modelo CR10X, da Campbell Sci.), localizado a 1/3 do comprimento do ambiente protegido, sendo medidas radiação solar global, temperatura e umidade relativa do ar. Para a medida da radiação, foi utilizado um piranômetro (modelo LI-200X, da LI-COR) a 1,5 m acima do dossel da cultura; para medir a temperatura e a umidade relativa do ar, foi utilizado um sensor (modelo HMP 35C da Campbell Sci) a 1,5 m do solo. As medições foram realizadas entre 6 e $18 \mathrm{~h}$, com intervalos de 30 minutos. No ambiente externo, foram utilizadas as observações efetuadas durante o período de realização do trabalho, na Estação Agrometeorológica da ESALQ/USP, situada a $500 \mathrm{~m}$ do local. Com os dados médios do intervalo de 30 minutos, foram realizadas correlações entre os valores dos elementos meteorológicos internos e externos ao longo do ciclo da cultura, por meio de regressão linear simples.

\section{RESULTADOS E DISCUSSÃO}

Os valores médios diários da radiação solar global medida no interior e exterior do ambiente protegido, ao longo do ciclo do meloeiro, bem como a análise de regressão, são apresentados na Figura 1. Os valores integrados durante o período de estudo das energias para radiação solar global interna (RsgI) totalizaram $1.353,14 \mathrm{MJ} \mathrm{m}^{-2}$ com média de $14,27 \mathrm{MJ} \mathrm{m}^{-2}$ dia $^{-1}$, enquanto para a radiação solar global externa (RsgE) foi de $1.853,14 \mathrm{MJ} \mathrm{m}^{-2}$ com média de $19,51 \mathrm{MJ} \mathrm{m}^{-2} \mathrm{dia}^{-1}$, sendo superior em $26,8 \%$ em relação à medida no interior do ambiente protegido. 
Observa-se que os valores de RsgI e RsgE apresentam grandes variações entre os dias, coincidindo com a variação de nebulosidade (Figura 1A). CARDOSO (2002), no período de 4-9 a 1512-2001, cultivando em estufa o mesmo híbrido de melão em Piracicaba - SP, encontrou RsgI média de 29,39 $\mathrm{MJ} \mathrm{m}^{-2} \mathrm{dia}^{-1}, 205,95 \%$ a mais do que registrado no presente trabalho, devido, possivelmente, às características de dimensões do ambiente protegido e à época de cultivo.

Para os dados obtidos, o modelo de regressão de melhor ajuste e mais compatível com o comportamento dos dados foi o modelo linear (Figura 1B), do tipo $y=0,7092 x+0,4388\left(R^{2}=0,9173\right)$. Verifica-se que aumentos da RsgE, aumentam também a RsgI; isso indica a possibilidade de controlar a quantidade de energia produzida em qualquer estado fenológico da cultura, mediante a utilização de material plástico e diferentes densidades que produzem reflexão e absorção dessa energia.

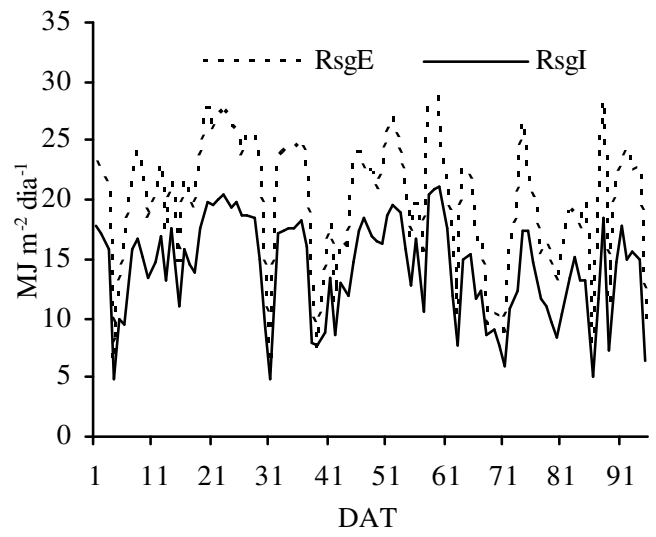

(A)
DAT

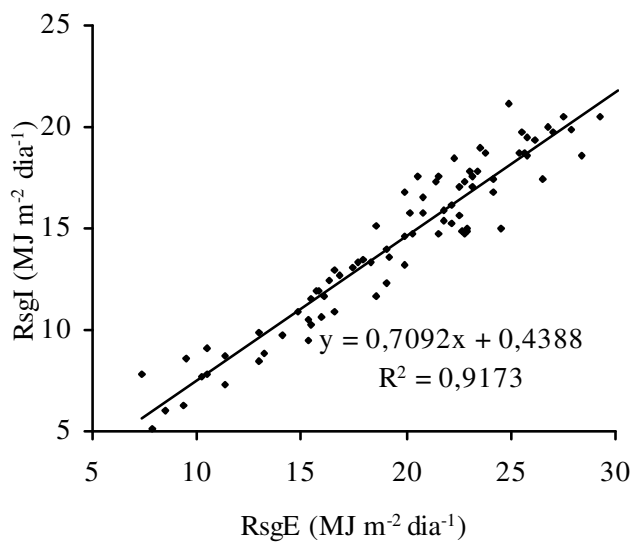

(B)

FIGURA 1. Radiação solar global diária no interior (RsgI) e exterior (RsgE) do ambiente protegido, em MJ m² dia $^{-1}$, ao longo do ciclo da cultura de melão (A) e correlação entre RsgI e RsgE (B).

Na Figura 2, estão apresentados os valores médios diários de temperatura no interior (TI) e exterior (TE) do ambiente protegido, e a análise de regressão respectiva.

Observa-se acréscimo de $0,99{ }^{\circ} \mathrm{C}$ no valor médio da temperatura do ambiente protegido em relação ao ambiente externo (Figura 2A), o que já era esperado devido à interrupção do processo convectivo pela cobertura plástica que impede a passagem do ar quente para o exterior; além disso, deve-se considerar o menor volume de ar a ser aquecido. A diferença de temperatura verificada entre os dois ambientes está próxima à encontrada por EVANGELISTA (1999), que foi de $1,6{ }^{\circ} \mathrm{C}$, e por SCATOLINI (1996), que tiveram diferença de $1,2^{\circ} \mathrm{C}$. De acordo com BURIOL et al. (1993), o valor médio de temperatura mínima do ar no interior de estufas cobertas com FEBD tende a ser igual ou ligeiramente superior, quando comparado com o ambiente externo. FARIAS et al. (1993) encontraram menores valores de temperaturas médias mínimas no interior do ambiente protegido, com variação diária entre os dois ambientes de $-1,0$ a $1,4^{\circ} \mathrm{C}$ a favor dos valores internos.

A relação entre os valores de TI e TE (Figura 2B) apresenta ajuste linear do tipo y =0,8798 $\mathrm{x}+$ 3,8913 ), com $\mathrm{R}^{2}=0,7624$, indicando a possibilidade de controlar a temperatura no interior do ambiente protegido fazendo manejo de abertura e fechamento das cortinas laterais e as janelas frontais em qualquer período fenológico da cultura, podendo evitar problemas com doenças, favorecidas pelo fenômeno de gutação das plantas, quando não existe renovação periódica do ar circundante. 


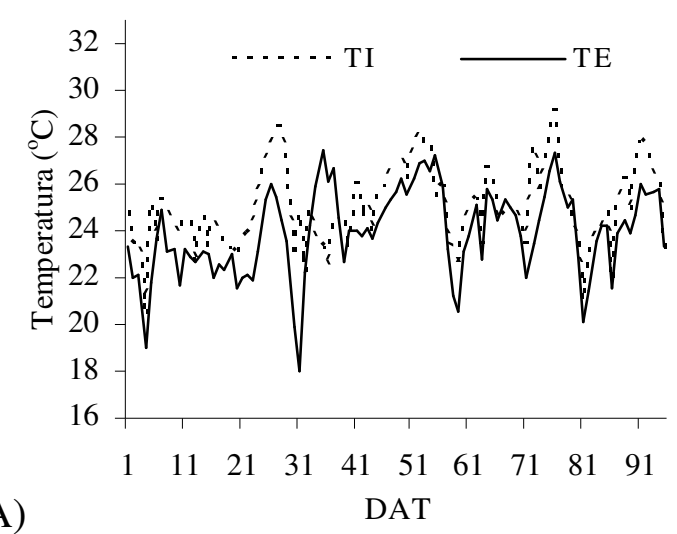

(A)

FIGURA 2. Temperatura média diária do ar no interior

Os valores de umidade relativa do ar no interior do ambiente protegido (URI) e do ambiente externo (URE) observados na Figura 3A demonstram menor amplitude da URI em relação ao ambiente externo, justificando o ajuste polinomial de $y=0,011 x^{2}-1,2087 x+97,411, R^{2}=0,6257$ (Figura 3B). Essa menor sensibilidade interna, provavelmente, está associada ao bloqueio dos ventos e conseqüente queda de arraste de moléculas de vapor d'água para fora do ambiente. No período de maior exigência hídrica, entre 30 e 60 dias após o transplantio (DAT), verificou-se que os valores médios de URI foram superiores aos de URE, provavelmente associado ao aumento de água evapotranspirada e à maior freqüência de irrigação. Resultados semelhantes foram encontrados por FARIAS et al. (1993) para a cultura feijão-de-vagem. Os valores médios da URI e da URE durante o ciclo foram de 71,17\% e de $74,25 \%$, respectivamente. De forma geral, a URI média foi 3,08\% menor em relação ao ambiente externo, próximos aos encontrados por EVANGELISTA (1999), que foi de 3,2\%.

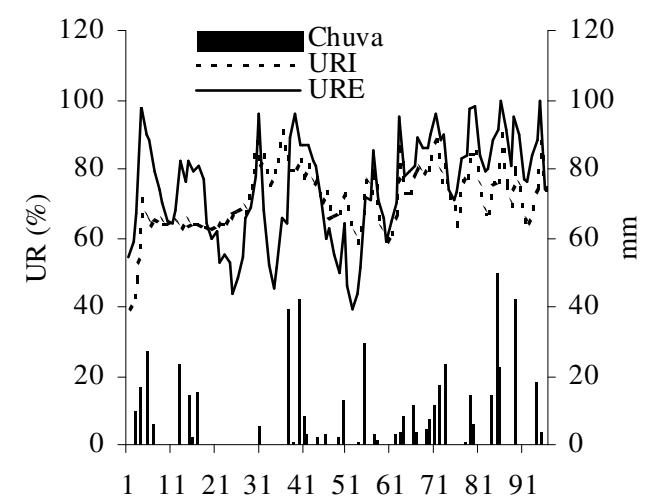

DAT

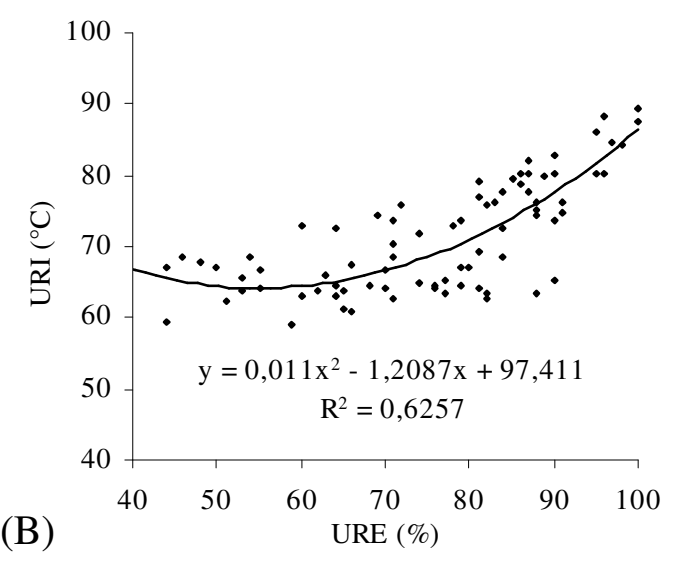

(A)

FIGURA 3. Valores de umidade relativa diária do ar no interior do ambiente protegido (URI) e ambiente externo (URE), e a precipitação diária e acumulada ao longo do ciclo do meloeiro (A), e a análise de regressão obtida para URE e URI (B).

Os elementos meteorológicos externos foram relacionados com a evaporação do minitanque (EV) no interior do ambiente protegido e são mostrados na Figura 4 (A e B). O melhor ajuste foi obtido considerando-se a umidade relativa do ar, seguido da radiação global $\left(\mathrm{R}^{2}=0,7061\right.$ e $\mathrm{R}^{2}=$ 0,6277, respectivamente). KIRDA (1994) encontrou relação linear entre a evaporação e a radiação com $\mathrm{R}^{2}=0,68$. $\mathrm{O}$ fato de a umidade relativa interna ser menos sensível às mudanças da umidade relativa externa, pouco afetou o ajuste; já a temperatura do ar não apresentou correlação com a evaporação, 
porém, é provável que a temperatura da folha tenha melhor relação, devido as leituras serem feitas na própria planta (microescala), registrando os mecanismos que produzem o gradiente de pressão de vapor dos estômatos.

(A)

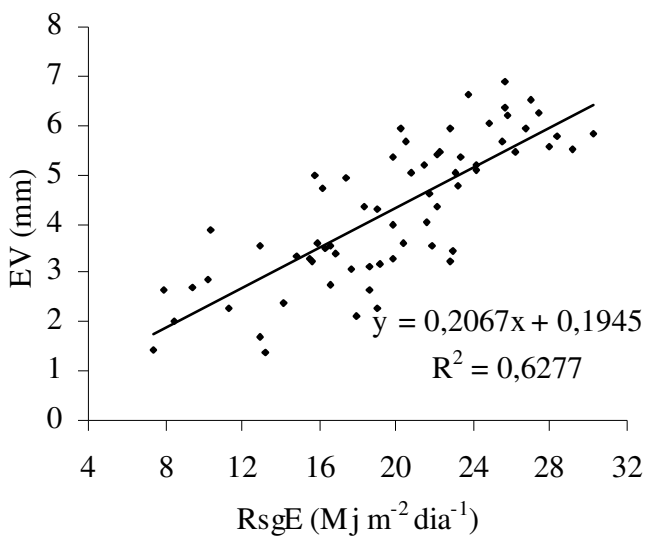

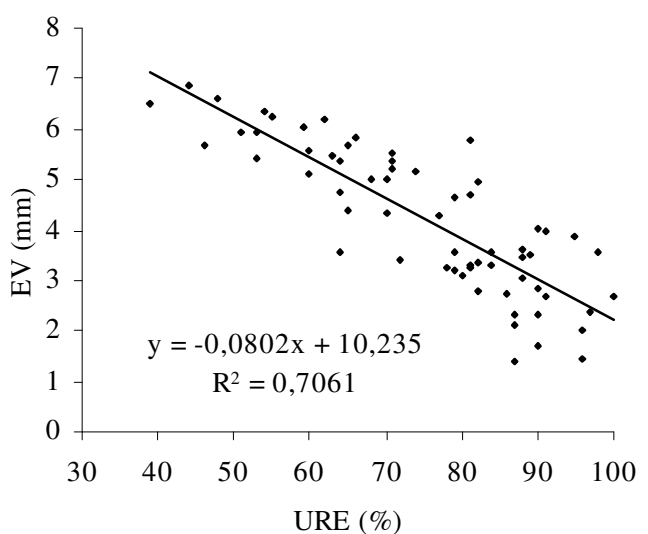

$\operatorname{URE}(\%)$

FIGURA 4. Relação entre a evaporação (EV) com a radiação global externa (RsgE) (A) e com a umidade relativa externa (URE) (B).

O consumo total de água ao longo do ciclo da cultua de melão para as condições do experimento foi de 279,60 mm. MONTEIRO (1995), trabalhando em condições semelhantes com a mesma cultura, encontrou consumo de $135 \mathrm{~mm}$. CARON \& HELDWEIN (2000), realizando trabalhos em condições idênticas, em 1996, 1997 e 1998, registraram consumos de 209,6; 166,8 e 159,1 mm, respectivamente. Por outro lado, quando se comparam estudos realizados referentes às necessidades hídricas da cultura a nível de campo, o consumo aumenta. Para DOORENBOS \& PRUITT (1976), o consumo de água para o cultivo do melão em campo, ao longo do seu ciclo de desenvolvimento (100 dias), resultou entre 400 e $600 \mathrm{~mm}$. HUDSON et al. (1981) recomendaram, para condições de campo, consumo mínimo de $500 \mathrm{~mm}$, próximo aos resultados encontrados por PEÑALBER (1997), que variaram entre 300 e $400 \mathrm{~mm}$ por ciclo.

\section{CONCLUSÕES}

A radiação solar global externa foi $26,8 \%$ maior do que a radiação solar global interna devido à reflexão e absorção do material da cobertura diminuírem a radiação solar global.

A umidade relativa interna manteve-se aproximadamente constante e menor do que a do meio externo, em função da não-interferência advectiva de correntes de ventos, embora os valores internos da umidade relativa tenham sido maiores do que os valores da umidade relativa externa para o período dos 30 aos 60 dias após o transplantio, em função da maior evapotranspiração da cultura e menor perda da água para o meio exterior.

A temperatura interna foi superior à temperatura externa em torno de $4 \%$, em função da interrupção do processo convectivo do meio interno com o meio externo, pois a cobertura plástica impede a passagem do ar quente do interior do ambiente protegido para o meio atmosférico.

Houve correlação entre os elementos meteorológicos observados no interior do ambiente protegido e no meio atmosférico, bem como correlação entre esses elementos externos e a evaporação interna, exceto para a temperatura do ar.

\section{REFERÊNCIAS}

BAR-YOSEF, B. Advances in fertigation. In: SPARKS, D.L. (Ed.). Advances in agronomy. New York: Academic Press, 1999. p.44-9. 
BURIOL, G.A.; STRECK, N.A.; PETRY, C. Transmissividade à radiação solar do polietileno da baixa densidade utilizado em estufas. Ciência Rural, Santa Maria, v.25, n 1, p.1-4, 1993.

CARDOSO, S.S. Dose de $\mathrm{CO}_{2}$ e de potássio aplicados através de irrigação no meloeiro rendilhado (Cucumis melo L.) cultivado em ambiente protegido. 2002. 101 f. Tese (Doutorado em Irrigação e Drenagem) - Escola Superior de Agricultura “Luiz de Queiroz”, Universidade de São Paulo, Piracicaba, 2002.

CARON, B.O.; HELDWEIN, A.B. Consumo d'água e coeficiente de cultura para o meloeiro cultivado em estufa plástica na primavera. Revista Brasileira de Agrometeorologia, Santa Maria, v.8, n.1, p.19$25,2000$.

DOORENBOS, J.; PRUITT, W.O. Las necesidades de agua de los cultivos. Roma: FAO, 1976. 194 p. (Boletim, 24).

EVANGELISTA, A.W.P. Avaliação de métodos de determinação da evapotranspiração, no interior da casa de vegetação. 1999. 79 f. Dissertação (Mestrado em Irrigação e Drenagem) - Universidade Federal de Lavras, Lavras, 1999.

FARIAS, J.R.B.; BERGAMASCHI, H.; MARTINS, S.R.; BERLATO, M.A. Efeito da cobertura plástica de estufa sobre a radiação solar. Revista Brasileira de Agrometeorologia, Santa Maria, v.1, n.1, p.31-6, 1993.

HUDSON, T.; HARTMANN, T.; FLOCKER, W.J. Growth, development and utilization of cultivated plants. In:__. Vegetable crops for fruits seeds. New York: Prentice-Hall, 1981. p.551-2.

JONES, H.G. Plants and microclimate. $2^{\text {nd }}$ ed. Cambridge: University Press, 1992. 428 p.

KIEHL, E.J. Fertilizantes orgânicos. São Paulo: Agronômica Ceres, 1985.147 p.

KIRDA, C.; ÇEVIK, B.; TÜLÜCÜ, K. A simple method to estimate the irrigation water requirement of greenhouse grown tomato. Acta Horticulturae, Wageningen, v.1, n.59, p.373-80, 1994.

MONTEIRO, S.B. Irrigação por gotejamento na cultura do melão em estufa e seu efeito na produção. 1995. 83 f. Dissertação (Mestrado em Energia na Agricultura) - Faculdade de Ciências Agronômicas, Universidade Estadual Paulista, Botucatu, 1995.

OLIVEIRA, M.R.V. O emprego de casas de vegetação no Brasil: Vantagens e desvantagens. Pesquisa Agropecuária Brasileira, Brasília, v.30, n.8, p.1049-60, 1995.

PEÑALBER, P.J. Cultivo del melón al aire libre. In: NAMESNY, A. Melones. Madrid: Ediciones de Horticultura, 1997. cap.7, p.58-65 (Compêndios de Horticultura, 10).

PEREIRA, A.R.; VILLA NOVA, N.A.; SEDIYMA, G.C. Evapo(transpi)ração. Piracicaba: FEALQ, 1997. 183 p.

RAIJ, B. van; CANTARELA, H.; QUAGGIO, J. A. et al. Recomendações de adubação e calagem para o Estado de São Paulo. Campinas: Instituto Agronômico de Campinas, 1986. 181 p. (Boletim Técnico, 100).

SCATOLINI, M.E. Estimativa da evapotranspiração da cultura de crisântemo em estufa a partir de elementos meteorológicos. 1996. 71 f. Dissertação (Mestrado em Irrigação e Drenagem) - Escola Superior de Agricultura “Luiz de Queiroz”, Universidade de São Paulo, Piracicaba, 1996.

SEEMAN, J. Greenhouse climate. In: . Agrometeorology. Berlin Heidelbery: Springer-Verlag, 1979. p.165-78. 